\title{
COMMON FIXED POINTS OF ONE-PARAMETER NONEXPANSIVE SEMIGROUPS IN STRICTLY CONVEX BANACH SPACES
}

TOMONARI SUZUKI

Received 20 December 2003; Accepted 10 July 2005

One of our main results is the following convergence theorem for one-parameter nonexpansive semigroups: let $C$ be a bounded closed convex subset of a Hilbert space $E$, and let $\left\{T(t): t \in \mathbb{R}_{+}\right\}$be a strongly continuous semigroup of nonexpansive mappings on $C$. Fix $u \in C$ and $t_{1}, t_{2} \in \mathbb{R}_{+}$with $t_{1}<t_{2}$. Define a sequence $\left\{x_{n}\right\}$ in $C$ by $x_{n}=\left(1-\alpha_{n}\right) /$ $\left(t_{2}-t_{1}\right) \int_{t_{1}}^{t_{2}} T(s) x_{n} d s+\alpha_{n} u$ for $n \in \mathbb{N}$, where $\left\{\alpha_{n}\right\}$ is a sequence in $(0,1)$ converging to 0 . Then $\left\{x_{n}\right\}$ converges strongly to a common fixed point of $\left\{T(t): t \in \mathbb{R}_{+}\right\}$.

Copyright (c) 2006 Tomonari Suzuki. This is an open access article distributed under the Creative Commons Attribution License, which permits unrestricted use, distribution, and reproduction in any medium, provided the original work is properly cited.

\section{Introduction}

Let $C$ be a closed convex subset of a Banach space $E$, and let $T$ be a nonexpansive mapping on $C$, that is, $\|T x-T y\| \leq\|x-y\|$ for all $x, y \in C$. We know that $T$ has a fixed point in the case that $E$ is uniformly convex and $C$ is bounded; see Browder [4], Göhde [10], and Kirk [15]. We denote by $F(T)$ the set of fixed points of $T$.

Let $\left\{T(t): t \in \mathbb{R}_{+}\right\}$be a strongly continuous semigroup of nonexpansive mappings (nonexpansive semigroup, in short) on a closed convex subset $C$ of a Banach space $E$, that is,

(i) for each $t \in \mathbb{R}_{+}, T(t)$ is a nonexpansive mapping on $C$;

(ii) $T(s+t)=T(s) \circ T(t)$ for all $s, t \in \mathbb{R}_{+}$;

(iii) for each $x \in C$, the mapping $t \mapsto T(t) x$ from $\mathbb{R}_{+}$into $C$ is strongly continuous.

We also know that $\left\{T(t): t \in \mathbb{R}_{+}\right\}$has a common fixed point in the case that $E$ is uniformly convex and $C$ is bounded; see Browder [4]. Bruck [7] prove the following theorem.

Theorem 1.1 (Bruck [7]). Suppose a closed convex subset $C$ of a Banach space has the fixed point property for nonexpansive mappings, and $C$ is either weakly compact, or bounded and separable. Then for any commuting family $S$ of nonexpansive mappings on $C$, the set of common fixed points of $S$ is a nonempty nonexpansive retract of $C$. 
This theorem yields that $\left\{T(t): t \in \mathbb{R}_{+}\right\}$has a common fixed point in the case that $C$ has the fixed point property, and that $C$ is weakly compact, or bounded and separable.

Several authors have studied about convergence theorems for nonexpansive semigroups; see $[1,2,13,16,19,21,22]$ and others. For example, the following theorem is a corollary of Theorem 8 in [19].

Theorem 1.2 (Shioji and Takahashi [19]). Let $C$ be a bounded closed convex subset of a Hilbert space E. Let $\left\{T(t): t \in \mathbb{R}_{+}\right\}$be a strongly continuous semigroup of nonexpansive mappings on $C$. Let $\left\{\alpha_{n}\right\}$ and $\left\{t_{n}\right\}$ be sequences of real numbers satisfying $0<\alpha_{n}<1, \lim _{n} \alpha_{n}$ $=0, t_{n}>0$ and $\lim _{n} t_{n}=\infty$. Fix $u \in C$ and define a sequence $\left\{x_{n}\right\}$ in $C$ by

$$
x_{n}=\frac{1-\alpha_{n}}{t_{n}} \int_{0}^{t_{n}} T(s) x_{n} d s+\alpha_{n} u
$$

for $n \in \mathbb{N}$. Then $\left\{x_{n}\right\}$ converges strongly to a common fixed point of $\left\{T(t): t \in \mathbb{R}_{+}\right\}$.

Also, Suzuki[21] proved the following theorem.

Theorem 1.3 (Suzuki [21]). Let E, $C,\left\{T(t): t \in \mathbb{R}_{+}\right\}$be as in Theorem 1.2. Let $\left\{\alpha_{n}\right\}$ and $\left\{t_{n}\right\}$ be sequences of real numbers satisfying $0<\alpha_{n}<1, t_{n}>0$ and $\lim _{n} t_{n}=\lim _{n} \alpha_{n} / t_{n}=0$. Fix $u \in C$ and define a sequence $\left\{x_{n}\right\}$ in $C$ by

$$
x_{n}=\left(1-\alpha_{n}\right) T\left(t_{n}\right) x_{n}+\alpha_{n} u
$$

for $n \in \mathbb{N}$. Then $\left\{x_{n}\right\}$ converges strongly to a common fixed point of $\left\{T(t): t \in \mathbb{R}_{+}\right\}$.

We note that in these theorems, real sequences $\left\{t_{n}\right\}$ converge to 0 and $\infty$. So, it is natural to study convergence theorems under the assumption that $\left\{t_{n}\right\}$ is a constant sequence. In this paper, motivated by Theorems 1.2 and 1.3 , we consider such type of convergence theorems to a common fixed point of $\left\{T(t): t \in \mathbb{R}_{+}\right\}$.

\section{Preliminaries}

Throughout this paper we denote by $\mathbb{R}$ the set of real numbers, by $\mathbb{R}_{+}$the set of nonnegative real numbers, and by $\mathbb{N}$ the set of positive integers. For a Banach space $E$, we also denote by $E^{*}$ the dual space of $E$.

We recall that a Banach space $E$ is called strictly convex if $\|x+y\| / 2<1$ for all $x, y \in E$ with $\|x\|=\|y\|=1$ and $x \neq y$. We know the following lemma.

Lemma 2.1. Let $E$ be a Banach space. Then the following are equivalent:

(i) $E$ is strictly convex;

(ii) $\|\lambda x+(1-\lambda) y\|<1$ for all $\lambda \in(0,1)$ and $x, y \in E$ with $\|x\|=\|y\|=1$ and $x \neq y$;

(iii) if $\|x\|=\|y\|=\|\lambda x+(1-\lambda) y\|$ for some $\lambda \in(0,1)$, then $x=y$.

A Banach space $E$ is called uniformly convex if for each $\varepsilon>0$, there exists $\delta>0$ such that $\|x+y\| / 2<1-\delta$ for all $x, y \in E$ with $\|x\|=\|y\|=1$ and $\|x-y\| \geq \varepsilon$. It is clear that a uniformly convex Banach space is strictly convex. The norm of $E$ is called Fréchet differentiable if for each $x \in E$ with $\|x\|=1, \lim _{t \rightarrow 0}(\|x+t y\|-\|x\|) / t$ exists and is attained uniformly in $y \in E$ with $\|y\|=1$. A Banach space $E$ is said to have the Opial property [17] 
if for each weakly convergent sequence $\left\{x_{n}\right\}$ in $E$ with weak limit $z, \liminf _{n}\left\|x_{n}-z\right\|<$ $\liminf _{n}\left\|x_{n}-y\right\|$ for all $y \in E$ with $y \neq z$. All Hilbert spaces, all finite dimensional Banach spaces and $\ell^{p}(1 \leq p<\infty)$ have the Opial property. Gossez and Lami Dozo[11] prove that every weakly compact convex subset of a Banach space with the Opial property has normal structure. We also know that every separable Banach space can be equivalently renormed so that it has the Opial property; see [23].

\section{Common fixed points}

In this section, we give our main results. The following proposition plays an important role in this paper.

Proposition 3.1. Let $C$ be a closed convex subset of a strictly convex Banach space E. Let $\tau_{\infty}>0$ and let $\left\{T(t): t \in\left[0, \tau_{\infty}\right)\right\}$ be a family of mappings on $C$ satisfying the following:

(i) for each $t \in\left[0, \tau_{\infty}\right), T(t)$ is nonexpansive;

(ii) there exists a strictly increasing sequence $\left\{\tau_{n}\right\}$ in $\left[0, \tau_{\infty}\right)$ such that $\tau_{1}=0,\left\{\tau_{n}\right\}$ converges to $\tau_{\infty}$, and mappings $t \mapsto T(t) x$ are weakly continuous on $\left[\tau_{n}, \tau_{n+1}\right)$ for all $x \in C$ and $n \in \mathbb{N}$.

Suppose that

$$
\bigcap_{t \in\left[0, \tau_{\infty}\right)} F(T(t)) \neq \varnothing .
$$

Then

$$
\bigcap_{t \in\left[0, \tau_{\infty}\right)} F(T(t))=F(S),
$$

where $S$ is a nonexpansive mapping on $C$ defined by

$$
S x=\frac{1}{\tau_{\infty}} \int_{0}^{\tau_{\infty}} T(s) x d s
$$

for all $x \in C$.

Remark 3.2. We do not assume $\{T(\cdot)\}$ is a nonexpansive semigroup.

Proof. Fix $f \in E^{*}$. Then the functions $t \mapsto f(T(t) x)$ from $\left[\tau_{n}, \tau_{n+1}\right)$ into $\mathbb{R}$ are continuous on $\left[\tau_{n}, \tau_{n+1}\right)$ for $x \in C$ and $n \in \mathbb{N}$. So, the functions $t \mapsto f(T(t) x)$ from $\left[0, \tau_{\infty}\right)$ into $\mathbb{R}$ are measurable for $x \in C$. We also have $\left\{T(t) x: t \in\left[0, \tau_{\infty}\right)\right\}$ is separable for each $x \in C$. Fix $w \in \bigcap_{t \in\left[0, \tau_{\infty}\right)} F(T(t))$. Since

$$
\begin{aligned}
\|T(t) x\| & =\|T(t) x\|-\|T(t) w\|+\|w\| \leq|T(t) x-T(t) w|+\|w\| \\
& \leq\|x-w\|+\|w\|,
\end{aligned}
$$

for $x \in C$ and $t \in\left[0, \tau_{\infty}\right)$, we have that the mappings $t \mapsto T(t) x$ are Bochner integrable for all $x \in C$ and hence $S$ is well-defined. Using the separation theorem, we can easily prove 
4 Nonexpansive semigroup in SC

that $S$ is a mapping on $C$. Since

$$
\begin{aligned}
\|S x-S y\| & =\mid \frac{1}{\tau_{\infty}} \int_{0}^{\tau_{\infty}}(T(s) x-T(s) y) d s \| \\
& \leq \frac{1}{\tau_{\infty}} \int_{0}^{\tau_{\infty}}\|T(s) x-T(s) y\| d s \\
& \leq \frac{1}{\tau_{\infty}} \int_{0}^{\tau_{\infty}}\|x-y\| d s=\|x-y\|
\end{aligned}
$$

for $x, y \in C, S$ is nonexpansive. Therefore $S$ is a nonexpansive mapping on $C$. It is obvious that $\bigcap_{t \in\left[0, \tau_{\infty}\right)} F(T(t)) \subset F(S)$. We assume that $z \in F(S) \backslash \bigcap_{t \in\left[0, \tau_{\infty}\right)} F(T(t))$. Then there exists $t_{1} \in\left[0, \tau_{\infty}\right)$ such that $T\left(t_{1}\right) z \neq z$. Fix $g \in E^{*}$ with

$$
\|g\|=1, \quad g\left(T\left(t_{1}\right) z-z\right)=\left\|T\left(t_{1}\right) z-z\right\| .
$$

For some $m \in \mathbb{N}, t_{1}$ belongs to $\left[\tau_{m}, \tau_{m+1}\right)$. From the assumption (ii), there exists $t_{2} \in$ $\left(t_{1}, \tau_{m+1}\right)$ such that

$$
g(T(t) z-z)>\frac{1}{2}\left\|T\left(t_{1}\right) z-z\right\|
$$

for all $t \in\left[t_{1}, t_{2}\right)$. Define nonexpansive mappings $S_{1}$ and $S_{2}$ on $C$ by

$$
\begin{aligned}
& S_{1} x=\frac{1}{t_{2}-t_{1}} \int_{t_{1}}^{t_{2}} T(s) x d s, \\
& S_{2} x=\frac{1}{\tau_{\infty}-t_{2}+t_{1}}\left(\int_{0}^{t_{1}} T(s) x d s+\int_{t_{2}}^{\tau_{\infty}} T(s) x d s\right)
\end{aligned}
$$

for all $x \in C$. We note that

$$
S x=\frac{t_{2}-t_{1}}{\tau_{\infty}} S_{1} x+\frac{\tau_{\infty}-t_{2}+t_{1}}{\tau_{\infty}} S_{2} x
$$

for all $x \in C$. We have

$$
\begin{aligned}
g\left(S_{1} z-S z\right) & =g\left(\frac{1}{t_{2}-t_{1}} \int_{t_{1}}^{t_{2}} T(s) z d s-z\right) \\
& =g\left(\frac{1}{t_{2}-t_{1}} \int_{t_{1}}^{t_{2}}(T(s) z-z) d s\right) \\
& =\frac{1}{t_{2}-t_{1}} \int_{t_{1}}^{t_{2}} g(T(s) z-z) d s \\
& \geq \frac{1}{t_{2}-t_{1}} \int_{t_{1}}^{t_{2}} \frac{1}{2}\left\|T\left(t_{1}\right) z-z\right\| d s \\
& =\frac{1}{2}\left\|T\left(t_{1}\right) z-z\right\|>0 .
\end{aligned}
$$

Hence

$$
g\left(S_{2} z-S z\right)=\frac{t_{2}-t_{1}}{\tau_{\infty}-t_{2}+t_{1}} g\left(S z-S_{1} z\right)<0
$$


Therefore $S_{1} z \neq S_{2} z$. Fix $w \in \bigcap_{t \in\left[0, \tau_{\infty}\right)} F(T(t))$. Then we note that $S_{1} w=S_{2} w=w$. We have

$$
\begin{aligned}
\|z-w\| & =\|S z-w\|=\left\|\frac{t_{2}-t_{1}}{\tau_{\infty}} S_{1} z+\frac{\tau_{\infty}-t_{2}+t_{1}}{\tau_{\infty}} S_{2} z-w\right\| \\
& \leq \frac{t_{2}-t_{1}}{\tau_{\infty}}\left\|S_{1} z-w\right\|+\frac{\tau_{\infty}-t_{2}+t_{1}}{\tau_{\infty}}\left\|S_{2} z-w\right\| \\
& =\frac{t_{2}-t_{1}}{\tau_{\infty}}\left\|S_{1} z-S_{1} w\right\|+\frac{\tau_{\infty}-t_{2}+t_{1}}{\tau_{\infty}}\left\|S_{2} z-S_{2} w\right\| \\
& \leq \frac{t_{2}-t_{1}}{\tau_{\infty}}\|z-w\|+\frac{\tau_{\infty}-t_{2}+t_{1}}{\tau_{\infty}}\|z-w\|=\|z-w\|
\end{aligned}
$$

and hence

$$
\|S z-w\|=\left\|S_{1} z-w\right\|=\left\|S_{2} z-w\right\|
$$

This contradicts the strict convexity of $E$. Therefore, $F(S) \subset \bigcap_{t \in\left[0, \tau_{\infty}\right)} F(T(t))$. This completes the proof.

As a direct consequence of Proposition 3.1, we can prove the following, which was proved by Bruck [6]; see also [20].

Corollary 3.3 (Bruck [6]). Let $C$ be a closed convex subset of a strictly convex Banach space E. Let $\left\{T_{n}: n \in \mathbb{N}\right\}$ be a sequence of nonexpansive mappings on $C$. Suppose $\bigcap_{n=1}^{\infty} F\left(T_{n}\right)$ is nonempty. Let $\left\{\alpha_{n}\right\}$ be a sequence of positive numbers with $\sum_{n=1}^{\infty} \alpha_{n}=1$. Define a nonexpansive mapping $S$ on $C$ by

$$
S x=\sum_{n=1}^{\infty} \alpha_{n} T_{n} x
$$

for $x \in C$. Then $F(S)=\bigcap_{n=1}^{\infty} F\left(T_{n}\right)$ holds.

Proof. Define a strictly increasing sequence $\left\{\tau_{n}\right\}$ in $[0,1)$ by $\tau_{1}=0$ and

$$
\tau_{n}=\sum_{k=1}^{n-1} \alpha_{k}
$$

for $n \in \mathbb{N}$ with $n \geq 2$. We note that $\lim _{n} \tau_{n}=1$. Define a family $\{T(t): t \in[0,1)\}$ of nonexpansive mappings as follows: If $\tau_{n} \leq t<\tau_{n+1}$, then

$$
T(t) x=T_{n} x
$$

for all $x \in C$. Then we note that

$$
S x=\sum_{n=1}^{\infty} \alpha_{n} T_{n} x=\sum_{n=1}^{\infty} \int_{\tau_{n}}^{\tau_{n+1}} T(s) x d s=\int_{0}^{1} T(s) x d s=\frac{1}{1} \int_{0}^{1} T(s) x d s
$$


6 Nonexpansive semigroup in SC

for $x \in C$ and

$$
\bigcap_{n=1}^{\infty} F\left(T_{n}\right)=\bigcap_{t \in[0,1)} F(T(t)) .
$$

So, by Proposition 3.1, we obtain the desired result.

As another direct consequence of Proposition 3.1, we obtain the following proposition.

Proposition 3.4. Let $C$ be a closed convex subset of a strictly convex Banach space E. Let $\tau>0$ and let $\{T(t): t \in[0, \tau)\}$ be a family of mappings on $C$ satisfying the following:

(i) for each $t \in[0, \tau), T(t)$ is nonexpansive;

(ii) mappings $t \mapsto T(t) x$ are weakly continuous on $[0, \tau)$ for all $x \in C$.

Suppose that

$$
\bigcap_{t \in[0, \tau)} F(T(t)) \neq \varnothing .
$$

Then

$$
\bigcap_{t \in[0, \tau)} F(T(t))=F(S)
$$

where $S$ is a nonexpansive mapping on $C$ defined by

$$
S x=\frac{1}{\tau} \int_{0}^{\tau} T(s) x d s
$$

for all $x \in C$.

Now, we prove one of our main results.

Theorem 3.5. Let $C$ be a closed convex subset of a strictly convex Banach space E and let $\left\{T(t): t \in \mathbb{R}_{+}\right\}$be a strongly continuous semigroup of nonexpansive mappings on $C$. Suppose that

$$
\bigcap_{t \in \mathbb{R}_{+}} F(T(t)) \neq \varnothing
$$

Fix $t_{1}, t_{2} \in \mathbb{R}_{+}$with $t_{1}<t_{2}$, and define a nonexpansive mapping $S$ on $C$ by

$$
S x=\frac{1}{t_{2}-t_{1}} \int_{t_{1}}^{t_{2}} T(s) x d s
$$

for all $x \in C$. Then

$$
\bigcap_{t \in \mathbb{R}_{+}} F(T(t))=F(S)
$$

holds. 
Proof. It is clear that $\bigcap_{t \in \mathbb{R}_{+}} F(T(t)) \subset F(S)$. Fix $w \in F(S)$. By Proposition 3.4, we have

$$
\bigcap_{t \in\left[t_{1}, t_{2}\right)} F(T(t))=F(S)
$$

So, $T(t) w=w$ for $t \in\left[t_{1}, t_{2}\right)$. Hence, for every $t \in\left[0,\left(t_{2}-t_{1}\right) / 2\right]$, we have

$$
T(t) w=T(t) \circ T\left(t_{1}\right) w=T\left(t+t_{1}\right) w=w .
$$

Let $t \in \mathbb{R}_{+}$be fixed. Then there exist $m \in \mathbb{N} \cup\{0\}$ and $u \in\left[0,\left(t_{2}-t_{1}\right) / 2\right)$ such that $t=$ $u+m\left(t_{2}-t_{1}\right) / 2$. We have

$$
T(t) w=T\left(u+m \frac{t_{2}-t_{1}}{2}\right) w=T(u) \circ T\left(\frac{t_{2}-t_{1}}{2}\right)^{m} w=T(u) w=w,
$$

where $T\left(\left(t_{2}-t_{1}\right) / 2\right)^{0}$ is the identity mapping on $C$. Therefore $w$ is a common fixed point of $\left\{T(t): t \in \mathbb{R}_{+}\right\}$. This completes the proof.

Similarly we can prove the following theorem.

Theorem 3.6. Let $C$ be a closed convex subset of a strictly convex Banach space E and let $\left\{\left\{T_{n}(t): t \in \mathbb{R}_{+}\right\}: n \in \mathbb{N}\right\}$ be a sequence of strongly continuous semigroups of nonexpansive mappings on $C$. Let $\left\{U_{n}: n \in \mathbb{N}\right\}$ be a sequence of nonexpansive mappings on $C$. Suppose that

$$
\bigcap_{n=1}^{\infty} \bigcap_{t \in \mathbb{R}_{+}} F\left(T_{n}(t)\right) \cap \bigcap_{n=1}^{\infty} F\left(U_{n}\right) \neq \varnothing .
$$

Let $\left\{t_{n}\right\},\left\{u_{n}\right\},\left\{\alpha_{n}\right\}$ and $\left\{\beta_{n}\right\}$ be real sequences such that $0 \leq t_{n}<u_{n}, \alpha_{n}>0$ and $\beta_{n}>0$ for all $n \in \mathbb{N}$, and $\sum_{n=1}^{\infty} \alpha_{n}+\sum_{n=1}^{\infty} \beta_{n}=1$. Define a nonexpansive mapping $S$ on $C$ by

$$
S x=\sum_{n=1}^{\infty} \frac{\alpha_{n}}{u_{n}-t_{n}} \int_{t_{n}}^{u_{n}} T_{n}(s) x d s+\sum_{n=1}^{\infty} \beta_{n} U_{n} x
$$

for all $x \in C$. Then

$$
\bigcap_{n=1}^{\infty} \bigcap_{t \in \mathbb{R}_{+}} F\left(T_{n}(t)\right) \cap \bigcap_{n=1}^{\infty} F\left(U_{n}\right)=F(S) .
$$

holds.

We recall that a closed convex subset $C$ of a Banach space $E$ is said to have the $f$ ixed point property for nonexpansive mappings ( $F P P$, in short) if for every bounded closed convex subset $D$ of $C$, every nonexpansive mapping on $D$ has a fixed point. So, by the results of Browder [4] and Göhde [10], every uniformly convex Banach space has FPP. Also, by Kirk's fixed point theorem [15], every weakly compact convex subset with normal structure has FPP.

As a direct consequence of Theorem 3.6, we obtain the following corollary. 
Corollary 3.7. Let $E, C,\left\{\left\{T_{n}(t): t \in \mathbb{R}_{+}\right\}: n \in \mathbb{N}\right\},\left\{U_{n}: n \in \mathbb{N}\right\},\left\{t_{n}\right\},\left\{u_{n}\right\},\left\{\alpha_{n}\right\}$, and $\left\{\beta_{n}\right\}$ be as in Theorem 3.6. Assume that $C$ is weakly compact and has FPP, and

$$
T_{m}(s) \circ T_{n}(t)=T_{n}(t) \circ T_{m}(s), \quad U_{m} \circ U_{n}=U_{n} \circ U_{m}, \quad U_{m} \circ T_{n}(t)=T_{n}(t) \circ U_{m}
$$

for all $s, t \in \mathbb{R}_{+}$and $m, n \in \mathbb{N}$. Define a nonexpansive mapping $S$ on $C$ as in Theorem 3.6. Then

$$
\bigcap_{n=1}^{\infty} \bigcap_{t \in \mathbb{R}_{+}} F\left(T_{n}(t)\right) \cap \bigcap_{n=1}^{\infty} F\left(U_{n}\right)=F(S) \neq \varnothing .
$$

holds.

\section{Convergence theorems}

Using Theorem 3.5, we can prove many convergence theorems to a common fixed point of nonexpansive semigroups. In this section, we state some of them.

From the result of Ishikawa [14], we obtain the following theorem see also Edelstein [8].

Theorem 4.1. Let $C$ be a compact convex subset of a strictly convex Banach space E. Let $\left\{T(t): t \in \mathbb{R}_{+}\right\}$be a strongly continuous semigroup of nonexpansive mappings on $C$. Fix $t_{1}, t_{2} \in \mathbb{R}_{+}$with $t_{1}<t_{2}$. Define a sequence $\left\{x_{n}\right\}$ in $C$ by $x_{1} \in C$ and

$$
x_{n+1}=\frac{\alpha_{n}}{t_{2}-t_{1}} \int_{t_{1}}^{t_{2}} T(s) x_{n} d s+\left(1-\alpha_{n}\right) x_{n}
$$

for $n \in \mathbb{N}$, where $\left\{\alpha_{n}\right\}$ is a sequence in $[0,1]$ satisfying $\sum_{n=1}^{\infty} \alpha_{n}=\infty$ and $\limsup _{n} \alpha_{n}<1$. Then $\left\{x_{n}\right\}$ converges strongly to a common fixed point of $\left\{T(t): t \in \mathbb{R}_{+}\right\}$.

From the results of Edelstein and O'Brien [9], and Reich [18], we obtain the following theorem.

Theorem 4.2. Let E be a Banach space. Suppose either of the following holds:

(i) $E$ is strictly convex and has the Opial property; or

(ii) $E$ is uniformly convex and its norm is Fréchet differentiable.

Let $C$ be a weakly compact convex subset of $E$, and let $\left\{T(t): t \in \mathbb{R}_{+}\right\}$be a strongly continuous semigroup of nonexpansive mappings on $C$. Fix $t_{1}, t_{2} \in \mathbb{R}_{+}$with $t_{1}<t_{2}$. Define a sequence $\left\{x_{n}\right\}$ in $C$ by $x_{1} \in C$ and

$$
x_{n+1}=\frac{\alpha}{t_{2}-t_{1}} \int_{t_{1}}^{t_{2}} T(s) x_{n} d s+(1-\alpha) x_{n}
$$

for $n \in \mathbb{N}$, where $\alpha$ is a constant number in $(0,1)$. Then $\left\{x_{n}\right\}$ converges weakly to a common fixed point of $\left\{T(t): t \in \mathbb{R}_{+}\right\}$.

We note that

$$
x \longmapsto(1-\alpha) T x+\alpha u
$$


is a contractive mapping if $T$ is a nonexpansive mapping and $\alpha \in(0,1)$. By the Banach contraction principle [3], such mappings have a unique fixed point. From the results of Browder [5], and Wittmann [24], we obtain the following theorem; see also [12]. Compare Theorem 4.3 with Theorems 1.2 and 1.3.

Theorem 4.3. Let $C$ be a bounded closed convex subset of a Hilbert space E, and let $\{T(t)$ : $\left.t \in \mathbb{R}_{+}\right\}$be a strongly continuous semigroup of nonexpansive mappings on $C$. Fix $u \in C$ and $t_{1}, t_{2} \in \mathbb{R}_{+}$with $t_{1}<t_{2}$. Define a sequence $\left\{x_{n}\right\}$ in $C$ by

$$
x_{n}=\frac{1-\alpha_{n}}{t_{2}-t_{1}} \int_{t_{1}}^{t_{2}} T(s) x_{n} d s+\alpha_{n} u
$$

for $n \in \mathbb{N}$, where $\left\{\alpha_{n}\right\}$ is a sequence in $(0,1)$ converging to 0 . Then $\left\{x_{n}\right\}$ converges strongly to a common fixed point of $\left\{T(t): t \in \mathbb{R}_{+}\right\}$.

Theorem 4.4. Let $E, C,\left\{T(t): t \in \mathbb{R}_{+}\right\}, u, t_{1}$ and $t_{2}$ be as in Theorem 4.3. Define a sequence $\left\{x_{n}\right\}$ in $C$ by $x_{1} \in C$ and

$$
x_{n+1}=\frac{1-\alpha_{n}}{t_{2}-t_{1}} \int_{t_{1}}^{t_{2}} T(s) x_{n} d s+\alpha_{n} u
$$

for $n \in \mathbb{N}$, where $\left\{\alpha_{n}\right\}$ is a sequence in $[0,1]$ satisfying the following:

$$
\lim _{n \rightarrow \infty} \alpha_{n}=0 ; \quad \sum_{n=1}^{\infty} \alpha_{n}=\infty ; \quad \sum_{n=1}^{\infty}\left|\alpha_{n+1}-\alpha_{n}\right|<\infty .
$$

Then $\left\{x_{n}\right\}$ converges strongly to a common fixed point of $\left\{T(t): t \in \mathbb{R}_{+}\right\}$.

\section{Acknowledgment}

The author is supported in part by Grants-in-Aid for Scientific Research from the Japanese Ministry of Education, Culture, Sports, Science and Technology.

\section{References}

[1] S. Atsushiba and W. Takahashi, Strong convergence theorems for one-parameter nonexpansive semigroups with compact domains, Fixed Point Theory and Applications (Y. J. Cho, J. K. Kim, and S. M. Kang, eds.), vol. 3, Nova Science, New York, 2002, pp. 15-31.

[2] J.-B. Baillon, Quelques propriétés de convergence asymptotique pour les semi-groupes de contractions impaires, Comptes Rendus de l'Académie des Sciences. Série A-B 283 (1976), no. 3, A75-A78.

[3] S. Banach, Sur les opérations dans les ensembles abstraits et leur application aux équations intégrales, Fundamenta Mathematicae 3 (1922), 133-181.

[4] F. E. Browder, Nonexpansive nonlinear operators in a Banach space, Proceedings of the Academy of Natural Sciences of Philadelphia U.S.A 54 (1965), 1041-1044.

[5] _ Convergence of approximants to fixed points of nonexpansive non-linear mappings in Banach spaces, Archive for Rational Mechanics and Analysis 24 (1967), no. 1, 82-90.

[6] R. E. Bruck Jr., Properties of fixed-point sets of nonexpansive mappings in Banach spaces, Transactions of the American Mathematical Society 179 (1973), 251-262. 
[7] __ A common fixed point theorem for a commuting family of nonexpansive mappings, Pacific Journal of Mathematics 53 (1974), no. 1, 59-71.

[8] M. Edelstein, A remark on a theorem of M. A. Krasnoselski, The American Mathematical Monthly 73 (1966), no. 5, 509-510.

[9] M. Edelstein and R. C. O'Brien, Nonexpansive mappings, asymptotic regularity and successive approximations, The Journal of the London Mathematical Society. Second Series 17 (1978), no. 3, $547-554$.

[10] D. Göhde, Zum Prinzip der kontraktiven Abbildung, Mathematische Nachrichten 30 (1965), 251-258.

[11] J.-P. Gossez and E. Lami Dozo, Some geometric properties related to the fixed point theory for nonexpansive mappings, Pacific Journal of Mathematics 40 (1972), no. 3, 565-573.

[12] B. Halpern, Fixed points of nonexpanding maps, American Mathematical Society. Bulletin 73 (1967), 957-961.

[13] N. Hirano, Nonlinear ergodic theorems and weak convergence theorems, Journal of the Mathematical Society of Japan 34 (1982), no. 1, 35-46.

[14] S. Ishikawa, Fixed points and iteration of a nonexpansive mapping in a Banach space, Proceedings of the American Mathematical Society 59 (1976), no. 1, 65-71.

[15] W. A. Kirk, A fixed point theorem for mappings which do not increase distances, The American Mathematical Monthly 72 (1965), no. 9, 1004-1006.

[16] I. Miyadera and K. Kobayasi, On the asymptotic behaviour of almost-orbits of nonlinear contraction semigroups in Banach spaces, Nonlinear Analysis 6 (1982), no. 4, 349-365.

[17] Z. Opial, Weak convergence of the sequence of successive approximations for nonexpansive mappings, American Mathematical Society. Bulletin 73 (1967), 591-597.

[18] S. Reich, Weak convergence theorems for nonexpansive mappings in Banach spaces, Journal of Mathematical Analysis and Applications 67 (1979), no. 2, 274-276.

[19] N. Shioji and W. Takahashi, Strong convergence theorems for asymptotically nonexpansive semigroups in Hilbert spaces, Nonlinear Analysis 34 (1998), no. 1, 87-99.

[20] T. Suzuki, Convergence theorems to common fixed points for infinite families of nonexpansive mappings in strictly convex Banach spaces, Nihonkai Mathematical Journal 14 (2003), no. 1, 43-54.

[21] _ On strong convergence to common fixed points of nonexpansive semigroups in Hilbert spaces, Proceedings of the American Mathematical Society 131 (2003), no. 7, 2133-2136.

[22] T. Suzuki and W. Takahashi, Strong convergence of Mann's type sequences for one-parameter nonexpansive semigroups in general Banach spaces, Journal of Nonlinear and Convex Analysis 5 (2004), no. 2, 209-216.

[23] D. van Dulst, Equivalent norms and the fixed point property for nonexpansive mappings, The Journal of the London Mathematical Society. Second Series 25 (1982), no. 1, 139-144.

[24] R. Wittmann, Approximation of fixed points of nonexpansive mappings, Archiv der Mathematik 58 (1992), no. 5, 486-491.

Tomonari Suzuki: Department of Mathematics, Kyushu Institute of Technology, Sensuicho, Tobata, Kitakyushu 804-8550, Japan

E-mail address: suzuki-t@mns.kyutech.ac.jp 


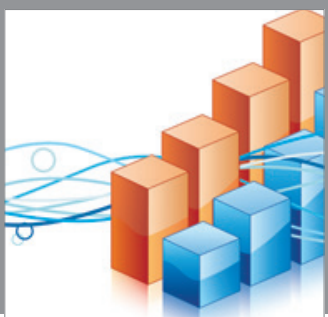

Advances in

Operations Research

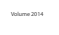

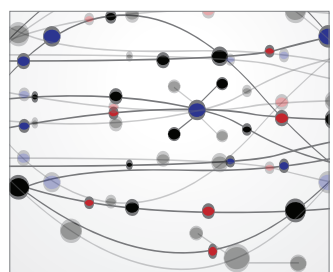

\section{The Scientific} World Journal
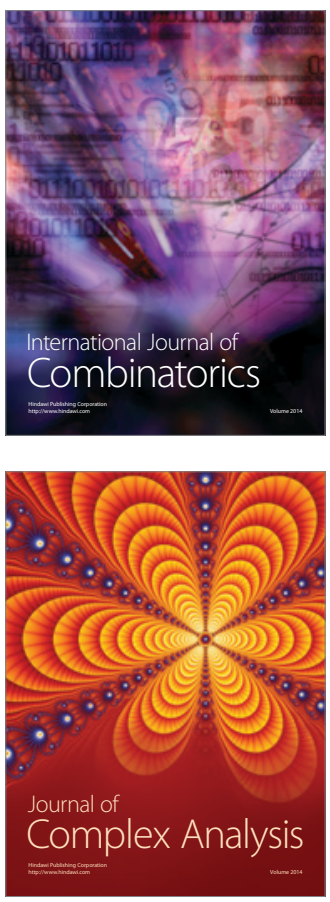

International Journal of

Mathematics and

Mathematical

Sciences
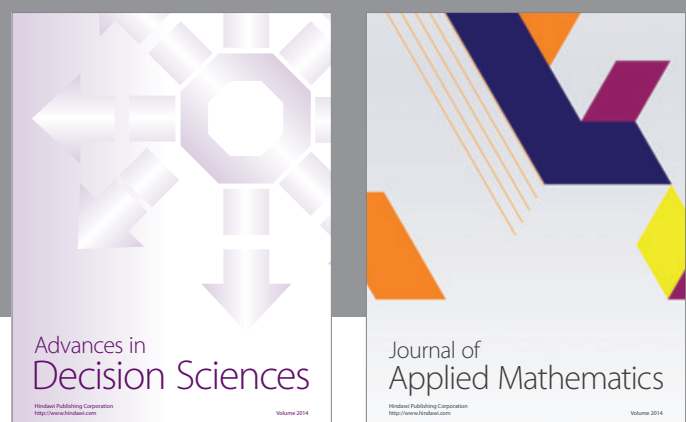

Journal of

Applied Mathematics
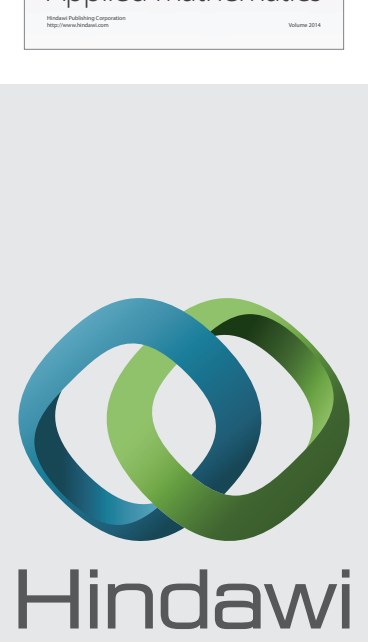

Submit your manuscripts at http://www.hindawi.com
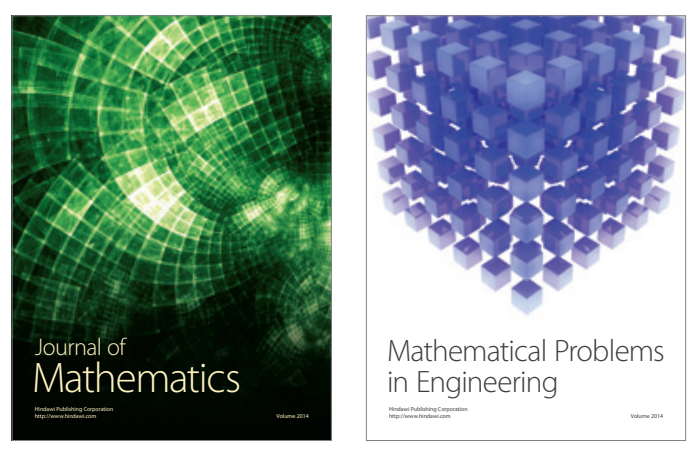

Mathematical Problems in Engineering
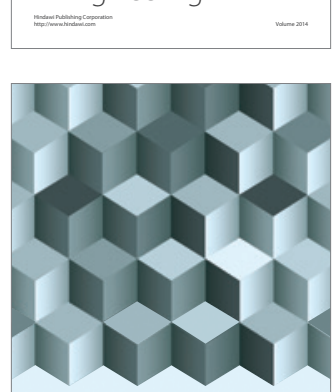

Journal of

Function Spaces
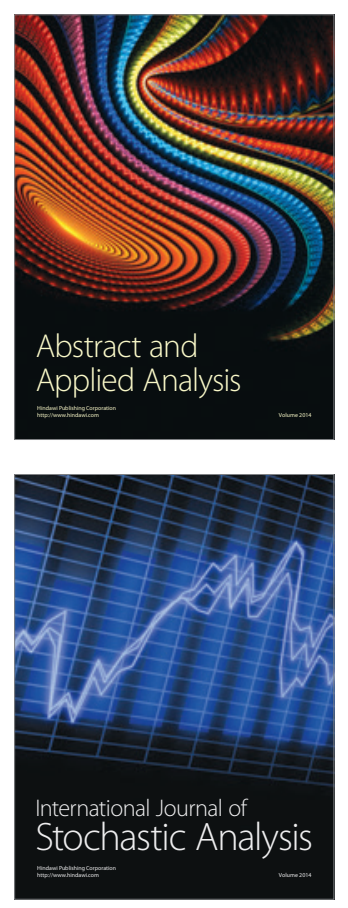

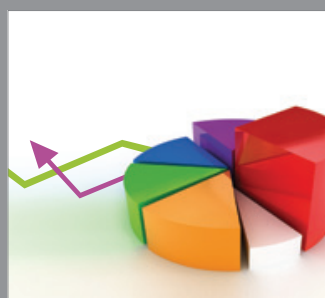

ournal of

Probability and Statistics

Promensencen
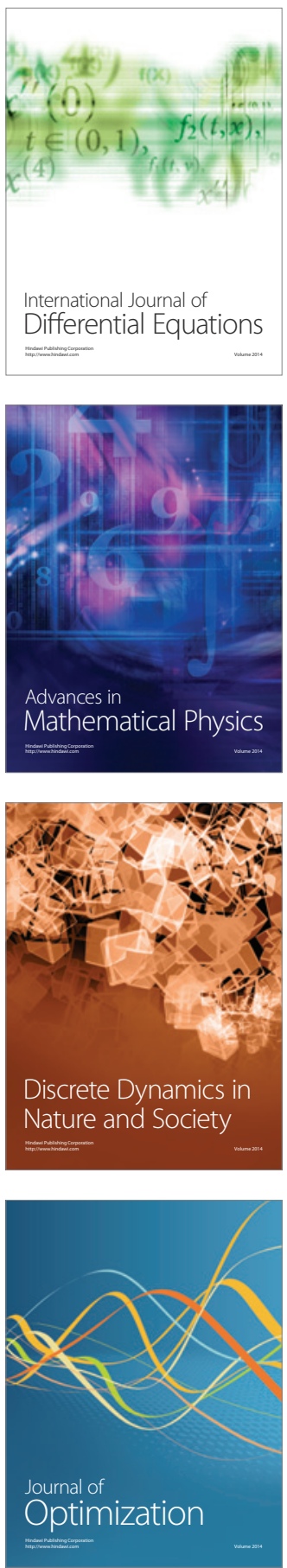